\title{
Importance of Strength Training of the Triceps Surae Muscles for Improvement of Walking Speed in Patients with Subacute Myelo-Optico-Neuropathy
}

\author{
Toshiaki Suzuki ${ }^{12^{*}}$, Sohei Yoshida ${ }^{1,2}$, Takayuki Nakayoshi ${ }^{2}$ \\ ${ }^{1}$ Graduate School of Kansai University of Health Sciences, Osaka, Japan \\ ${ }^{2}$ Research Centre of Neurological Diseases, Kansai University of Health Sciences, Osaka, Japan \\ *Corresponding author: Toshiaki Suzuki, Graduate School of Kansai University of Health Sciences, Osaka, Japan; \\ E-mail: suzuki@kansai.ac.jp
}

\begin{abstract}
Two patients with subacute myelo-optico-neuropathy (SMON) were evaluated by using the functional reach test (FRT) in the standing position, and by measuring the gait speed for $10 \mathrm{~m}$ before and after strength training of the triceps surae muscles through calf-raise training in the standing position. The reach distance in FRT and the walking speed after strength training of the triceps surae muscles through calf raising were improved compared with those before the calf-raise training in both patients. The results of this study suggested that strength training of the triceps surae muscles improves not only the walking ability but also the balance function in patients with SMON.
\end{abstract}

Received Date: March 3, 2017

Accepted Date: March 10, 2017

Published Date: March 16, 2017

Citation: Suzuki, T., et al. Importance of Strength Training of the Triceps Surae Muscles for Improvement of Walking Speed in Patients with Subacute Myelo-Optico-Neuropathy. (2017) Lett Health Biol Sci 2(1): 48- 51.

DOI: $10.15436 / 2475-6245.17 .014$

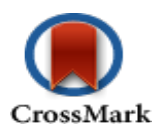

\section{Introduction}

Subacute myelo-optico-neuropathy (SMON) is a disease caused by clioquinol intoxication and is characterized by subacute onset of sensory and motor disturbance in the lower extremities, as well as visual impairment after the appearance of abdominal symptoms ${ }^{[1,2]}$. In Japan, the incidence of SMON peaked in the decade from 1960 to $1970^{[3]}$. Currently, there are more than 2000 patients with SMON, and these patients present with a number of serious neurological symptoms. Some studies have reported that activities of daily living (ADL) are limited in patients with SMON owing to their neurological symptoms $\mathrm{s}^{[, 5]}$. The status of patients with SMON in terms of ADL is variable: bed-ridden, dependent ADL, or independent ADL.

Among patients with SMON with independent ADL, many are not satisfied with their walking speed. We have observed that increasing the speed of walking was possible by improving the dorsiflexion movement of the ankle joint through strength training of the triceps surae muscles. In this study, we investigated the effect of strength training of the triceps surae muscle by using calf raising in two patients who could inde- pendently perform ADL but had a slow walking speed.

\section{Case Reports}

The subjects of this study were two patients (case A: a 79-year-old woman; case B: 74-year-oldwoman) with SMON. The goal of rehabilitation was improvement of the speed of gait in the two patients with independent ADL. The two patients performed the functional reach test (FRT) in the standing position, and their gait speed for $10 \mathrm{~m}$ was measured. The methods of FRT were as follows: (i) moving forward by using only dorsiflexion of the ankle joint in the standing position with $90^{\circ}$ flexion of both shoulder joints (FRT with the ankle method), and (ii) moving forward in the patient's chosen manner in the standing position with $90^{\circ}$ flexion of both shoulder joints (FRT with the free method) (Figures 1 and 2). The distance of reach was measured from standing to the maximum forward posture. The gait speed for $10 \mathrm{~m}$ was the fastest in three trials with free walking. 
Figure 1: Functional reach test (FRT) with two different methods (case A).

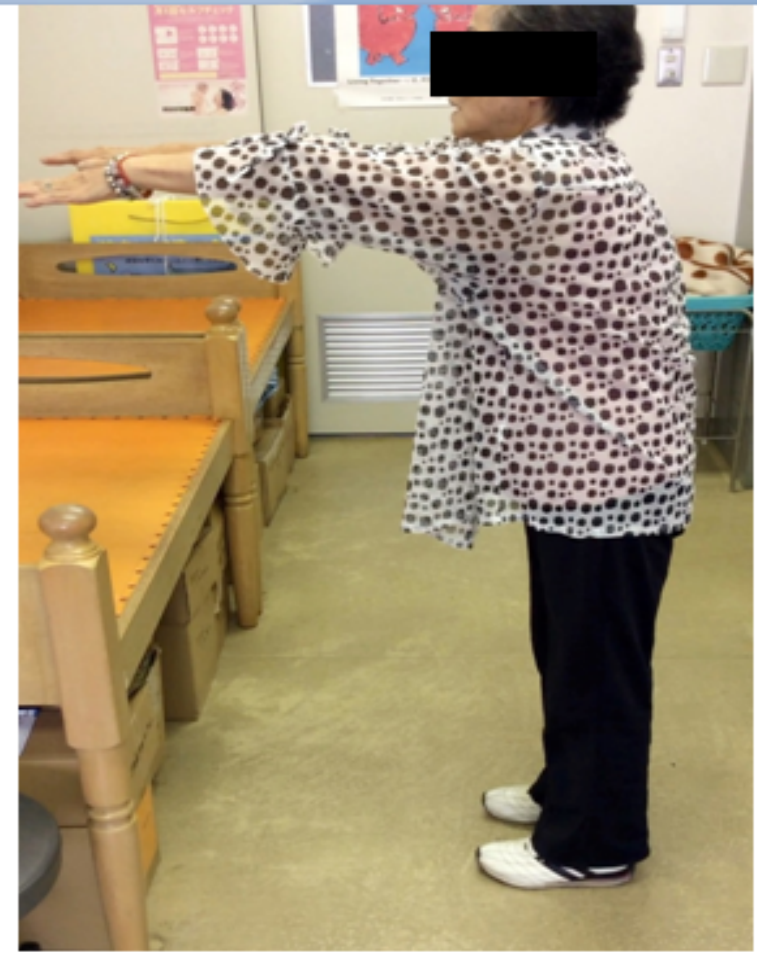

FRT with the ankle method

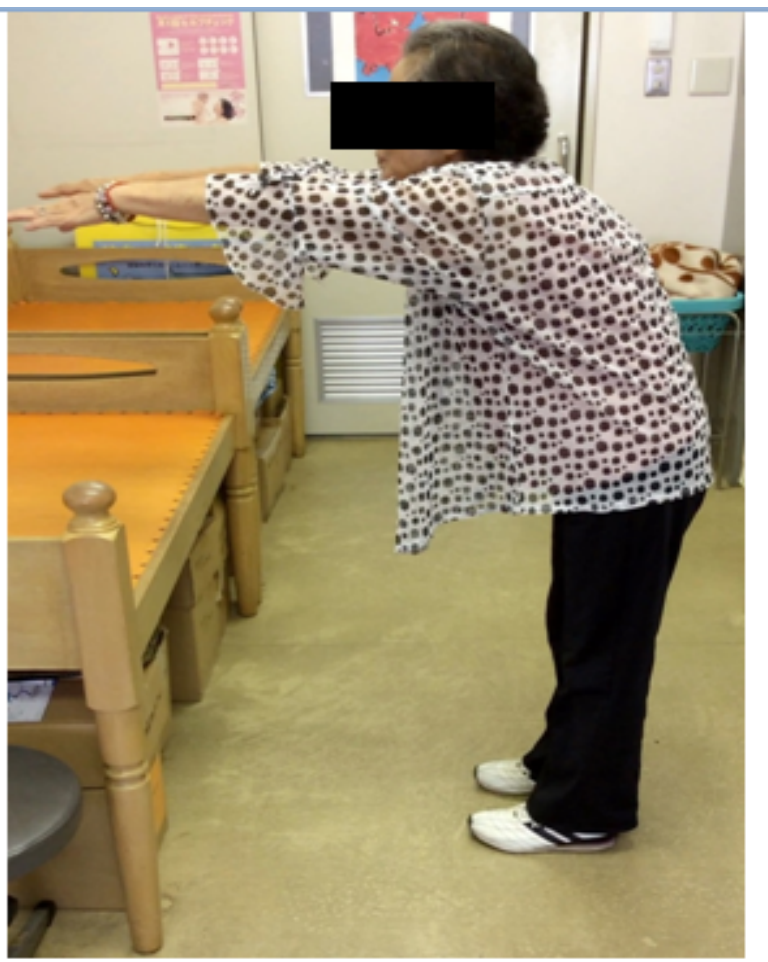

FRT with the free method

Figure 2: Functional reach test (FRT) with two different methods (case B).

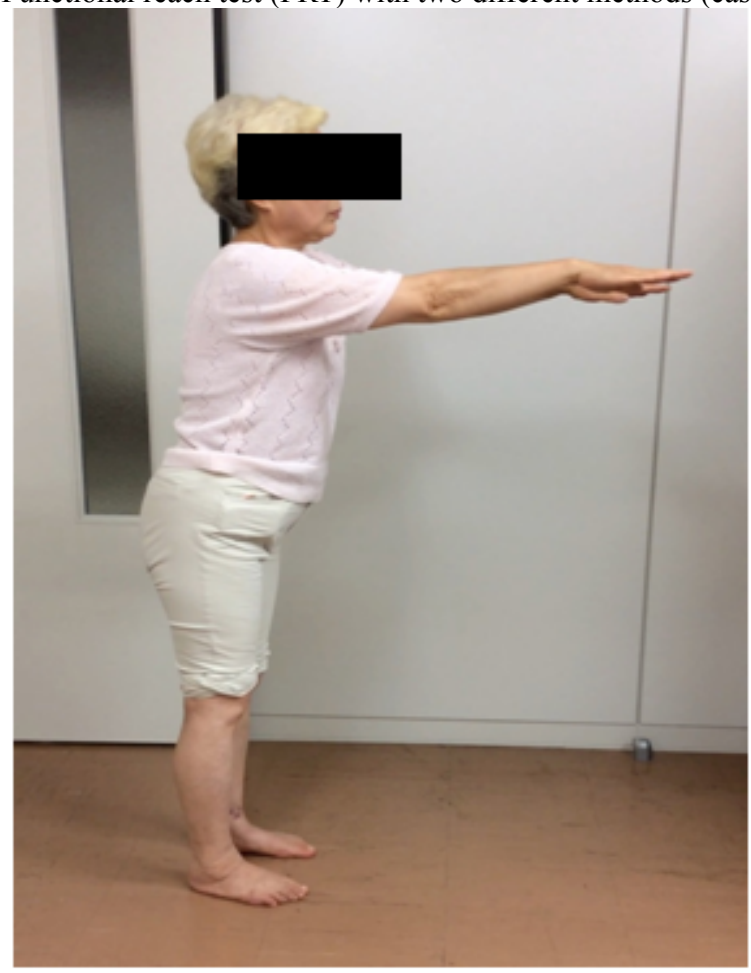

FRT with the ankle method

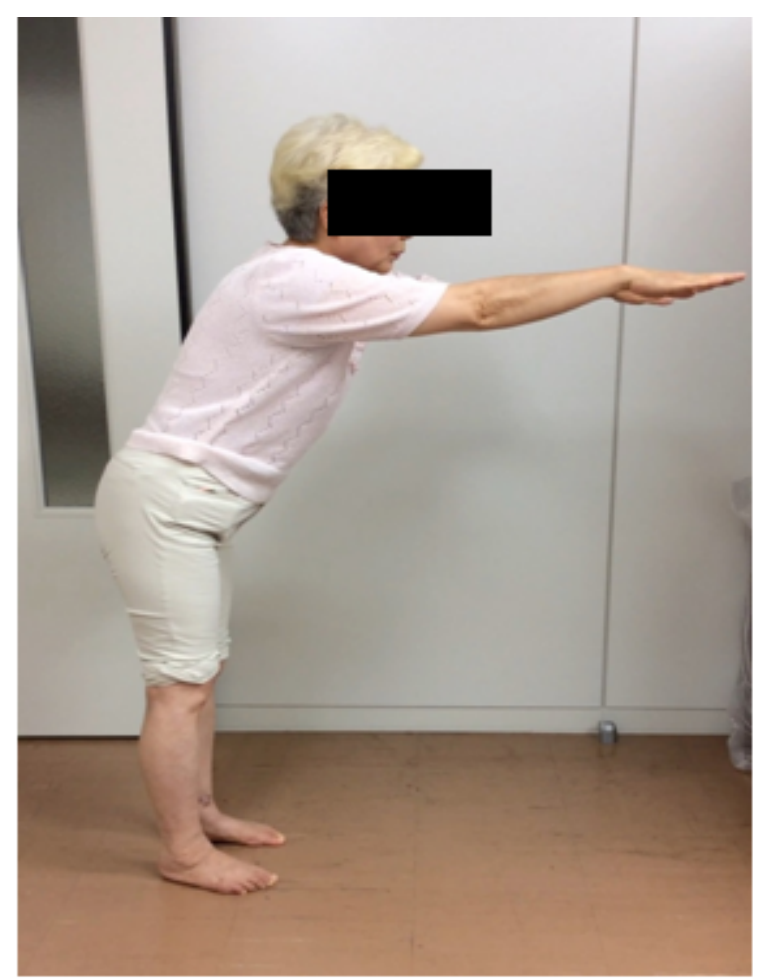

FRT with the free method

Next, calf-raise training in the standing position was done for strength training of the triceps surae muscles (Figure 3). The specific methods were as follows: (i) standing with wall support by using both arms; (ii) performing calf raising while standing, with maximum plantar flexion of the ankle joints maintained for $5 \mathrm{~s}$; and (iii) slowly moving from the calf-raised position to the starting standing position. This training was done three times in a row. After the calf-raise training, the functional reach and walking speed were tested again. We investigated the effect of calf-raise training on the changes in functional reach and walking speed. 


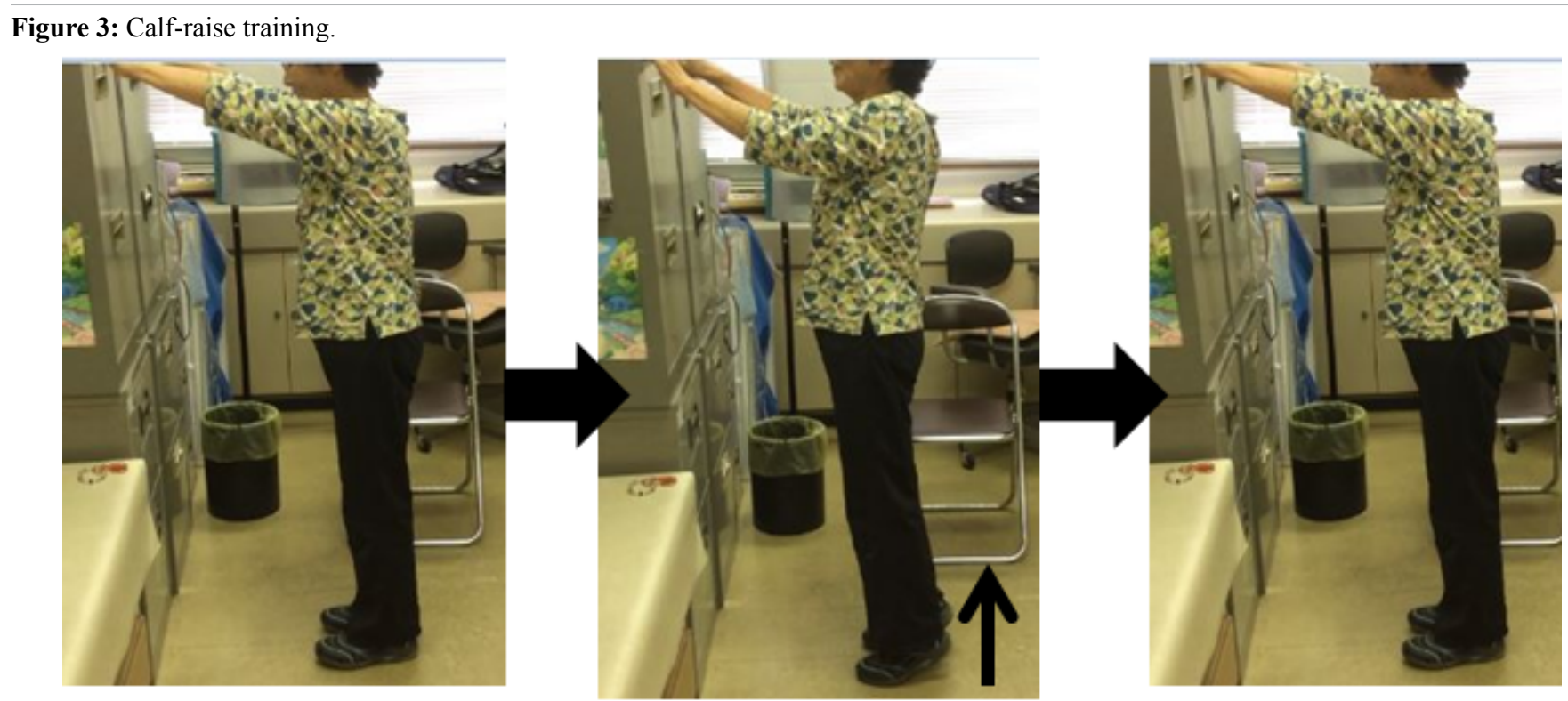

\section{Standing}

Calf raise

\section{(position kept for $5 \mathrm{~s}$ )}

\section{Results}

The distance of reach in FRT with the ankle method and that in FRT with the free method after the calf-raise training were improved compared with those before the calf-raise training in both patients (Figure 4). The speed of walking after calf-raise training was also improved slightly, faster by about $2 \mathrm{~s}$, than that before the calf-raise training.

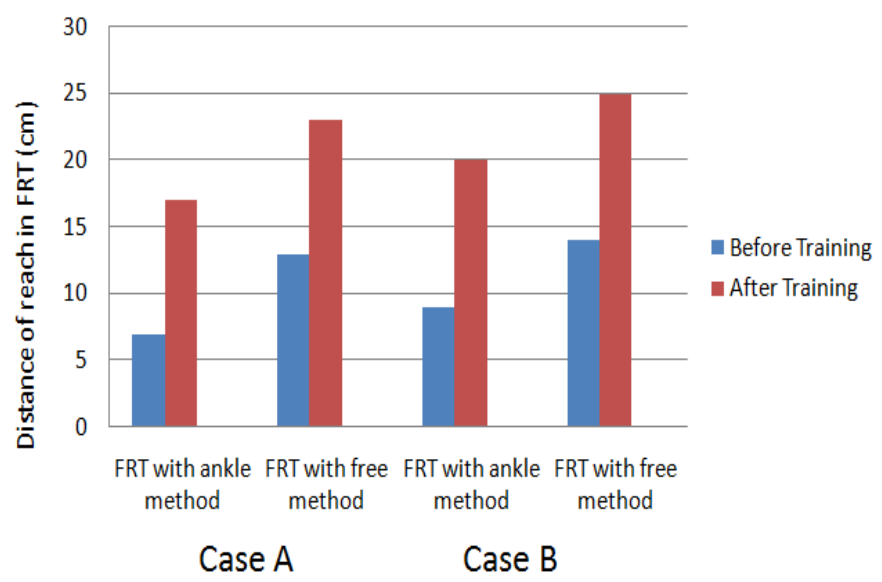

Figure 4: Distance of reach in the functional reach test (FRT) before and after calf-raise training.

\section{Discussion}

The function of ankle movement is very important in increasing the speed of walking. In the stance phase, anterior movement is required with dorsi-flexion of the ankle joint and with weight bearing on the sole of the feet. The muscles used for dorsiflexion of the ankle joint in the stance phase are the triceps surae muscles, not the tibialis anterior muscle. The ankle joints perform dorsiflexion movements with the triceps surae muscles to stop the anterior shifting of the lower leg in the stance phase. Honeine, et $\mathrm{al}^{[6]}$ reported that the duration of the stance phase proved to be highly correlated with the duration of triceps surae muscle activity.

In this study, we selected calf-raise training while standing as a strength training exercise for the triceps surae muscles. Calf-raise training is an easy method for patients and can be used in self-training. The important aspect of calf-raise training is that the speed of training is solely, especially the movement from the calf-raised position while standing to the starting standing position.

The functional reach distance in the standing position and the walking speed were used to determine the effect of calf-raise training. FRT is a typical evaluation tool for standing balance, introduced by Duncan et al ${ }^{[7]}$. The specific method of FRT by Duncan et $\mathrm{al}^{[7]}$ involves measurement of the distance from the standing position with $90^{\circ}$ flexion of the shoulder joint in the dominant side to the maximum anterior shifting in the standing position. We observed that the motion pattern during FRT involved dorsiflexion of the ankle joint with a faster speed; however, it also involved flexion of the hip joint and trunk with a slower walking speed.

In this study, two different methods of FRT were used: FRT with the ankle method as the test of the stability of the stance phase and FRT with the free method as the test of balance ability in the standing position. The functional reach distance in both FRT methods after strength training of the triceps surae muscle through calf raising was improved. From these results, calf-raise training is very important for improving walking ability, especially walking speed and balance ability, in patients with SMON. 


\section{Conclusion}

Two patients (case A: a 79-year-old woman; case B: a 74-year-old woman) with SMON were tested by using the FRT in the standing position, and their gait speed for $10 \mathrm{~m}$ was measured before and after strength training of the triceps surae muscles through calf-raise training in the standing position. The distance of reach in FRT and the speed of walking after strength training of the triceps surae muscles through calf-raise training were improved compared with those before calf-raise training in both patients. The results of this study suggest that strength training of the triceps surae muscles improves not only the walking ability but also the balance function in patients with SMON.

Acknowledgment: This work was supported in part by a Health and Labor Sciences Research Grant for Research on Intractable Diseases from The Ministry of Health and Welfare of Japan.

\section{References}

1. Tsubaki, T., Honma, Y., Hoshi, M. Neurological syndrome associated with clioquinol. (1971) Lancet 1(7701): 696-697.

Pubmed I Crossref I Others

2. Sobue, I., Ando, K., Iida, M., et al. Myeloneuropathy with abdominal disorders in Japan: a clinical study of 752 cases. (1971) Neurology 21(2): 168-173.

Pubmed I Crossref I Others

3. Nakae, K., Yamamoto, S., Shigematsu, I., et al. Relation between subacute myelo-optic neuropathy (S.M.O.N.) and clioquinol: nationwide survey. (1973) Lancet 1(7796): 171-173.

Pubmed I Crossref I Others

4. Konagaya, M., Matsumoto, A., Takase, S., et al. Clinical analysis of longstanding subacute myelo-optico-neuropathy: sequelae of clioquinol at 32 years after its ban. (2004) J Neurol Sci 218(1-2): 85-90.

Pubmed I Crossref I Others

5. Nagayoshi, M., Takahashi, M., Saeki, S., et al. Disability and lifestyle of subacute myelo-optico-neuropathy and elderly persons living at home: a comparison of the Barthel Index score and the Frenchay Activities Index score. (2007) J UOEH 29(4): 407-415.

Pubmed I Others

6. Honeine, J.L., Schieppati, M., Gagey, O., et al. By counteracting gravity, triceps surae sets both kinematics and kinetics of gait. (2014) Physiol Rep 2(2): e00229.

Pubmed I Crossref I Others

7. Duncan, P.W., Weiner, D.K., Chandler, J., et al. Functional reach: anew clinical measure of valance. (1990) J Gerontol 45(6): 192-197.

Pubmed I Crossref
Ommega Online Publishers

Journal Name: Letters In Health and Biological Sciences

Journal Short Name: Lett Health Biol Sci
ISSN no: 2475-6245

E-mail: healthandbiosciences@ommegaonline.com

Website: www.ommegaonline.org 